Original Research Paper

\title{
Assessment of Therapeutic Effects of Platelet-Rich Plasma in Knee Osteoarthritis: Possible Role of Inflammatory Cytokines
}

\author{
${ }^{1}$ Mohammed H. Hassan, ${ }^{2,3}$ Sawsan Abuhamdah, ${ }^{4}$ Tahia H. Saleem, ${ }^{5}$ Elsayed Said, \\ ${ }^{1}$ Nehal Ashraf Zaki, ${ }^{6}$ Ghada M. Abdelrazek, ${ }^{7}$ Safaa Y. Salim and ${ }^{5}$ Hamdy Tammam \\ ${ }^{1}$ Departments of Medical Biochemistry, Faculty of Medicine, South Valley University, Qena 83523, Egypt \\ ${ }^{2}$ College of Pharmacy, Al Ain University, Abu Dhabi, United Arab Emirates \\ ${ }^{3}$ Department of Biopharmaceutics and Clinical Pharmacy, Faculty of Pharmacy, The University of Jordan, Amman, Jordan \\ ${ }^{4}$ Department of Medical Biochemistry, Faculty of Medicine, Assiut University, Assiut, Egypt \\ ${ }^{5}$ Department of Orthopaedic Surgery, Faculty of Medicine, South Valley University, Qena 83523, Egypt \\ ${ }^{6}$ Department of Diagnostic Radiology, Faculty of Medicine, South Valley University, Qena 83523, Egypt \\ ${ }^{7}$ Department of Pharmacology, Faculty of Medicine, Assiut University, Assiut, Egypt
}

\section{Article history}

Received: 25-04-2021

Revised: 22-05-221

Accepted: 26-06-2021

Corresponding Author: Mohammed H. Hassan Departments of Medical Biochemistry, Faculty of Medicine, South Valley University, Qena 83523, Egypt Email:

Mohammedhosnyhassaan@yahoo.com mohammedhosnyhassaan@med.svu.edu.eg

\begin{abstract}
Osteoarthritis (OA) is a multifactorial disease that commonly affects the knee. Tumor Necrosis Factor- $\alpha(\mathrm{TNF}-\alpha)$ regulates inflammation during OA. Macrophage Migration Inhibitory Factor (MIF) may be involved in the pathophysiology of arthritis. Platelet-Rich Plasma (PRP) may reduce pain associated with OA. The present study assessed the possible therapeutic effects of PRP in patients with OA of the knee with varying severities. A prospective study was performed in 90 patients diagnosed with mild (30 cases), moderate (30 cases) and severe (30 cases) knee OA. Three Intra-Articular (IA) injections of PRP were administrated, 2 weeks a part, were received. Pain score and MRI Osteoarthritis Knee Score (MOAKS) were assessed. Serial synovial fluid cytokine assays to measure Tumor Necrosis Factor- $\alpha$ (TNF- $\alpha$ ) and macrophage Migration Inhibitory Factor (MIF) were performed using commercially available ELISA kits. The assays were performed pre-injection (S1), 2 weeks after the first IA injection (S2) and 2 weeks after the second IA injection (S3) for all patients. The mean values of pain score and synovial TNF- $\alpha$ and MIF levels were significantly higher (S1, pre-injection) among severe OA than among those with either mild or moderate cases, $\mathrm{p}<0.05$ for all. Pain score and synovial TNF- $\alpha$ and MIF levels at S3 were significantly lower in those with mild, moderate and severe OA than the corresponding S1 values, $(\mathrm{p}<0.05$ for all $)$. There was significant improvement in synovitis in both mild and moderate cases, $(\mathrm{p}<0.05$ for both). The IA injection of PRP reduces synovial fluid TNF- $\alpha$ and MIF levels significantly and exhibit significant therapeutic effects on synovitis by reducing inflammatory cytokine levels and bone marrow lesions primarily for mild knee OA and to a lesser extent for moderate cases.
\end{abstract}

Keywords: Platelet Rich Plasma, Knee Osteoarthritis, MRI Osteoarthritis Knee Score, Pain Score, Synovial Fluid, Tumor Necrosis Factor- $\alpha$, Macrophage Migration Inhibitory Factor

\section{Introduction}

Osteoarthritis (OA) is a disease primarily affects the knee. It is defined as a progressive loss of joint function and pain from the gradual deterioration of the articular cartilage (Kennedy et al., 2018). Unfortunately, it is a common malady because of the frequent use and stress of the knee joint, which causes a painful condition including OA (Richebé et al., 2018).

OA was thought to be primarily a degenerative disease of the cartilage; however, the latest research indicates that $\mathrm{OA}$ has a multifactorial cause involving 
numerous factors, including trauma, mechanical forces, inflammation, biochemical reactions and metabolic derangements (Ayhan et al., 2014). The role of inflammation is not well understood and there is an ongoing debate to whether the inflammatory reaction triggers changes in $\mathrm{OA}$ or weather the inflammation is secondary to changes in OA (Ayhan et al., 2014).

Tumor Necrosis Factor (TNF)- $\alpha$ expression is associated with the progression of OA. TNF- $\alpha$ has been shown to be capable of regulating inflammation in an OA rat model by down regulating the Phosphoinositide 3Kinase/protein Kinase B (PI3K/AKT) signaling pathway in synovial fibroblasts (Li et al., 2018).

Macrophage Migration Inhibitory Factor (MIF) is a pro-inflammatory cytokine produced by macrophages that may contribute to arthritis pathophysiology by promoting inflammation and angiogenesis (LlamasCovarrubias et al., 2013).

Current approaches to OA treatment have integrated the use of biologicals that mediate the inflammatory process (Kennedy et al., 2018). Platelet-Rich Plasma (PRP) is increasingly to influence tissue regulation because it contains growth factors and high platelet levels that reduce OA-related pain (Sampson et al., 2010; Halpern et al., 2013). PRP is obtained by whole blood centrifugation, yielding a highly concentrated product containing platelets. The $\alpha$-granules within the concentrated platelet solution contain growth factors and proteins that are vital to the coagulation cascade (Nurden, 2011). PRP is thought to influence the degeneration of cartilage by altering autophagy in chondrocytes. Aging cartilage gradually loses its reversible quiescence and its ability to self-renew (Chakkalakal et al., 2012).

In this study we used clinical and radiological scores to determine the potential therapeutic effects of PRP in patients with varying severities of knee OA. In addition, biochemical assays of TNF- $\alpha$ and MIF levels in synovial fluid were performed and their correlations with both pain and radiological scores were assessed among these patients.

\section{Patients and Methods}

\section{Study Design and Participants}

This prospective study was conducted on 90 patients with knee OA, recruited from Orthopedic Outpatient Clinics, at Qena University Hospital-South Valley University- Egypt, from February 1st, 2019 to January 31st, 2020. Based on radiological assessments, the patients were categorized into three groups (mild, moderate and severe OA) according to OA severity using MRI Osteoarthritis Knee Score (MOAKS). Each group consisted of 30 patients.

The exclusion criteria were as follows: Polyarticular disease; knee arthroscopy in the previous year; HA or steroid IA penetration in the preceding 3 months; history of infectious disease and autoimmune disorders such as diabetes, rheumatoid arthritis, hematologic diseases (coagulopathy), serious cardiovascular diseases, infections or immunodepression; anticoagulant therapy or an anti-aggregating agent; use of nonsteroidal antiinflammatory drugs 2 weeks prior to blood collection; and $<10 \mathrm{~g} / \mathrm{dL}$ of hemoglobin (Taniguchi et al., 2018).

\section{Clinical Assessments}

A detailed medical history and thorough clinical examination from every subject included age, gender, weight, height, calculation of Body Mass Index (BMI) $\left(\mathrm{kg} / \mathrm{m}^{2}\right)$. The BMI was classified as underweight (BMI $\left.<18.5 \mathrm{~kg} / \mathrm{m}^{2}\right)$, normal weight $\left(18.5-24.9 \mathrm{~kg} / \mathrm{m}^{2}\right)$, overweight $\left(25-29.9 \mathrm{~kg} / \mathrm{m}^{2}\right)$, obesity class 1 (30-34.9 $\left.\mathrm{kg} / \mathrm{m}^{2}\right)$, obesity class $2\left(35-39.9 \mathrm{~kg} / \mathrm{m}^{2}\right)$, extreme obesity class $3\left(>40 \mathrm{~kg} / \mathrm{m}^{2}\right)$ (Dwyer et al., 2000). Local examinations of both knee joints and assessments of knee joint pain for each patient were conducting using the Visual Analog Scale (VAS) score (Katz and Melzack, 1999). OA diagnosis was based on visual identification of eburnation on the articular surfaces of the distal femur, proximal tibia, or patella, right or left. Eburnation is a sclerotic, ivory-like subchondral bone reaction that occurs from bone-on-bone contact at sites exposed to advanced cartilage erosion (Rogers and Dieppe, 2003; Pritzker et al., 2006). The VAS is a common tool that uses a $10 \mathrm{~cm}$ scale to measure pain intensity, where $0=$ no pain and $10=$ unable to move. Pain score assessments were performed before the beginning PRP therapy and 2 weeks after the second PRP IA injection.

\section{Radiological Assessments of Severity of Knee OA}

MRI Osteoarthritis Knee Score was used to assess the severity of OA (Hunter et al., 2011). Briefly, the following assessments were used for both bone marrow abnormality and the patella-femoral cartilage volume: Grade I (mild if the lesion involved $<33 \%$ of subregional volume); grade II (moderate if the lesion involved 33-66\% of subregional volume) and grade III (severe if the lesion involved $>66 \%$ of subregional volume). Synovitis was categorized as follows: Grade 1 (mild or small-fluid continuous in the retropatellar space); grade 2 (moderate or medium - slight convexity of the suprapatellar bursa) and grade 3 (severe or large-evidence of capsular distension). A meniscal desintegrity score of 0-3 applied for the amount of extrusion at four locations: Medial meniscus (medial and anterior extrusion) and lateral meniscus (medial and anterior extrusion).

\section{Biochemical Workup}

PRP preparation: The PRP used for IA injection was prepared under completely aseptic conditions. To avoid the effect of food intake on purified PRP, patients were instructed to fast for $4 \mathrm{~h}$ on the day of injection before blood collection. Approximately $10 \mathrm{~mL}$ of venous blood was drawn from the antecubital vein using an aseptic technique in an effort to avoid irritation and trauma to the 
platelets. Anti-coagulated blood was obtained in five extraction tubes $(2 \mathrm{~mL}$ each), containing sodium citrate. The tubes were then centrifuged at $2100 \mathrm{rpm}$ at room temperature for $8 \mathrm{~min}$ to separate the citrated plasma from the buffy coat and the residual Red Blood Cells (RBCs) (Taniguchi et al., 2018). Using a pipette, the PRP situated just above the selectively precipitated RBCs but not including the buffy coat was aspirated carefully from each tube. In each affected joint, 5-mL PRP samples (from the five blood collection tubes) were used for IA injection (Dwyer et al., 2000). For each intra-articular injection new fresh PRP samples were made from the patients.

TNF- $\alpha$ and MIF were measured in synovial fluid samples using commercially available ELISA kits obtained from Chongqing Biospes, China (Catalog No.: BYEK3327-48T and BYEK3015) and a microplate ELISA Reader (EMR 500, USA) according to the manufactures' instructions. To extract cell debris, synovial fluid samples were immediately centrifuged at $3000 \mathrm{rpm}$ for $15 \mathrm{~min}$ and the supernatant was aliquoted into $1-\mathrm{mL}$ cryotubes and frozen at $-80^{\circ} \mathrm{C}$ until use. The assays were performed for the synovial fluid samples at three points: The first sample (S1) was pre-injection; the second Sample (S2) was 2 weeks after the first injection and the third Sample (S3) was 2 weeks after the second injection.

\section{Procedure and Timing of IA PRP Injections}

The patients were placed in a supine position with a 20-degree flexion of the knee. Under aseptic conditions, a 21-gauge needle was used to inject $5 \mathrm{~mL}$ of PRP into the suprapatellar knee joint pouch, using the superolateral method with ultrasound guidance. Local anesthetics were not used. After the injection, patients were instructed to refrain from physical exercise for at least $24 \mathrm{~h}$ but no restriction was specified regarding activities of daily living. Three IAPRP injections were administered at 2week intervals (Fig.1). The same physician who selected and tested the participants administered the injections (Taniguchi et al., 2018).

\section{Statistical Analyses}

IBM SPSS Statistics v.22 software was used for data analysis. The Mean \pm Standard Deviation (SD), median and inter-quartile range were used for expression of quantitative data, were as the number and percentage were used for qualitative data. The Kolmogro-Smirnov and Shapiro-Wilk normality tests were used. Independent sample T-tests were used to compare normally distributed quantitative variables between two groups, while MannWhitney Test used for abnormally distributed variables. One way-ANOVA was for comparison between more than two quantitative data. Post Hoc test Least Significance Difference (LSD) was used for multiple comparisons between different quantitative variables. For qualitative variables, chi-squared $\left(\chi^{2}\right)$ and Fisher's exact tests were used. Pearson's correlation coefficient (r) test was used to correlate data. $\mathrm{P}$ values $<0.05$ were considered statistically significant.

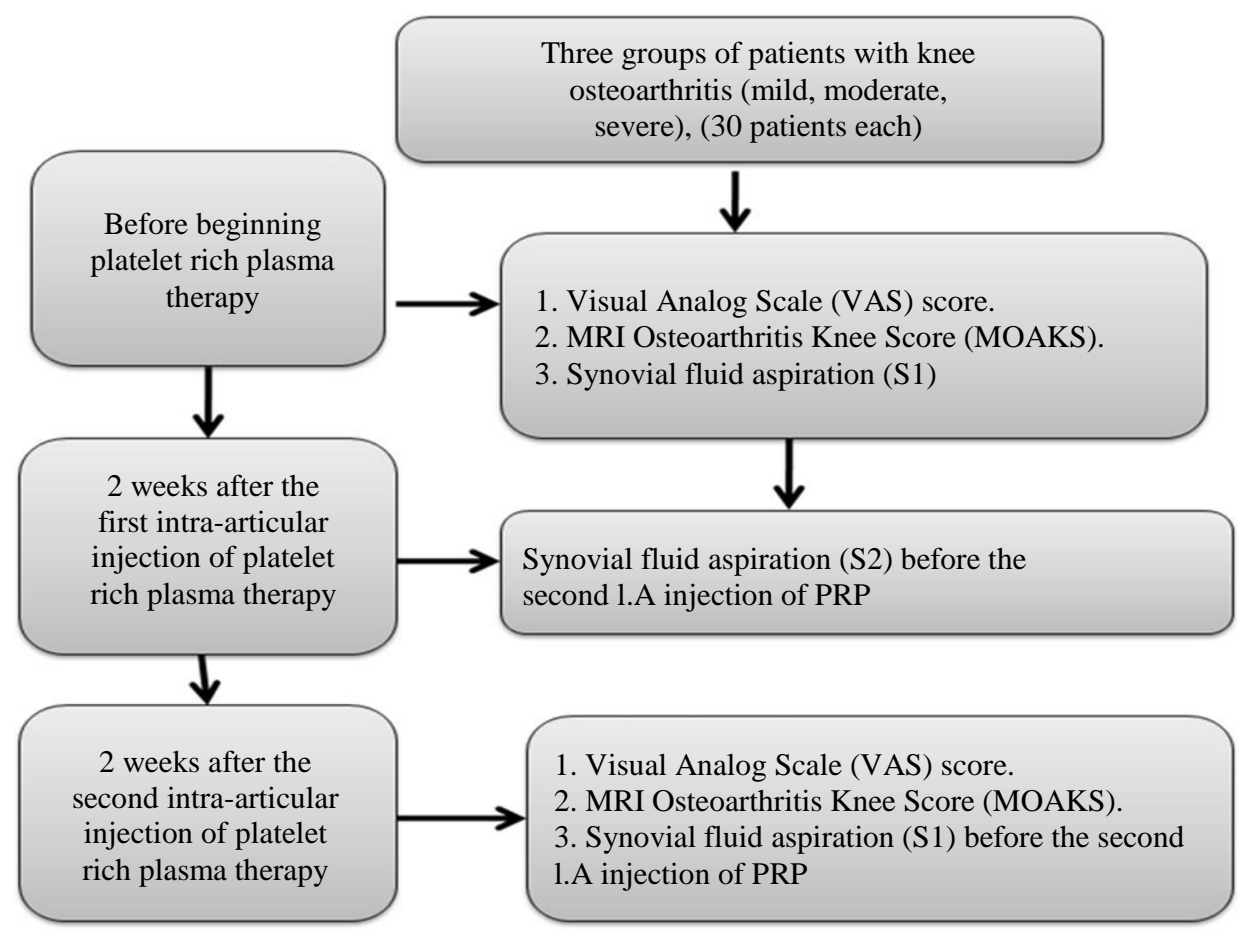

Fig. 1: Scheme for the study design. S1, S2 and S3 synovial fluid samples were analyzed biochemically for proinflammatory cytokines (Tumor Necrosis Factor- $\alpha$ and Macrophage Migration Inhibitory factor) 


\section{Results}

\section{Demographic and Clinical Characteristics of Study Participants}

The current study included 90 patients with knee OA [9 males $(10 \%)$ and $81(90 \%)$ females], with female-to-male ratio of 9:1. The subjects' mean \pm SD age was $45.5 \pm 10.6$ years with an age range of 25-65 years. The mean \pm SD BMI was $31.9 \pm 2.9 \mathrm{~kg} / \mathrm{m}^{2}$ with a range of $23-37 \mathrm{~kg} / \mathrm{m}^{2}$. The mean duration of knee OA was $28.8 \pm 17.5$ months with a range of 3-60 months, (Table1). With respect to the BMI, 7 patients $(7.7 \%)$ were categorized as overweight, 69 patients $(76.6 \%)$ as obesity class 1 and 14 patients $(15.5 \%)$ as obesity class 2 .

All patients had unilateral knee OA. The patients were categorized into three groups based on the severity of OA:30 patients (6 males and 24 females) had mild knee OA (33.3\%), 30 patients (30 females) had moderate severity (33.3\%) and 30 patients ( 3 males and 27 females) had severe OA (33.3\%). There were no significant differences with respect to the mean age $(46.8 \pm 10.2, \quad 46 \pm 12.3$ and $43.6 \pm 10.1$ years, respectively), BMI $(31 \pm 2.4,32.1 \pm 2.1$ and $32.6 \pm 3.9$, respectively), or sex $\mathrm{p}>0.05$ for all, (Table1).

Serial Synovial Fluid Levels of TNF- $\alpha$ as Regards Time of IA PRP Injection and Severity of Knee OA

The mean synovial TNF- $\alpha$ values were significantly higher (S1, pre-injection) in patients with severe OA $(144.8 \pm 13.6 \mathrm{pg} / \mathrm{mL})$ than in those with either mild $(35.5 \pm 7.4 \mathrm{pg} / \mathrm{mL})$ or moderate OA $(97.3 \pm 31.7 \mathrm{pg} / \mathrm{mL})$, $\mathrm{p}<0.05$ for all (Fig. 2A). Significantly lower synovial TNF- $\alpha$ levels were observed 2 weeks after the second IA injection of PRP (S3) in patients with mild, moderate or severe knee OA $(9.3 \pm 5.4,44.8 \pm 11.5$ and $59.7 \pm 3.9$, respectively) when compared with both the pre-injection synovial fluid levels (35.5 $\pm 7.4,97.3 \pm 31.7$ and 144.8 \pm 13.6 , respectively) and levels 2 weeks after the first injection (S2) $(17.8 \pm 7.5,64.6 \pm 15.3$ and $82.4 \pm 4.3$, respectively), $\mathrm{p}<0.05$ for all (Table2).

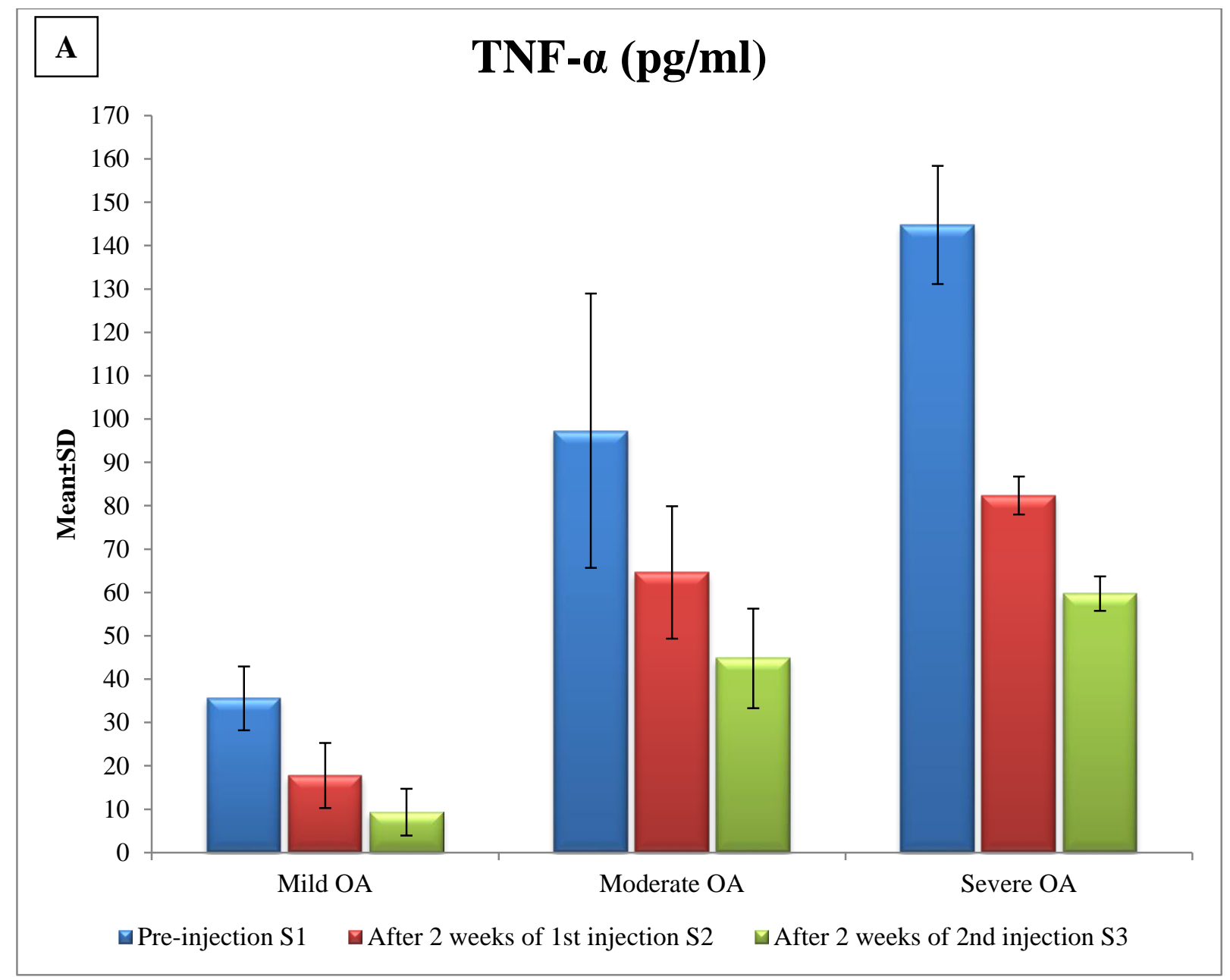




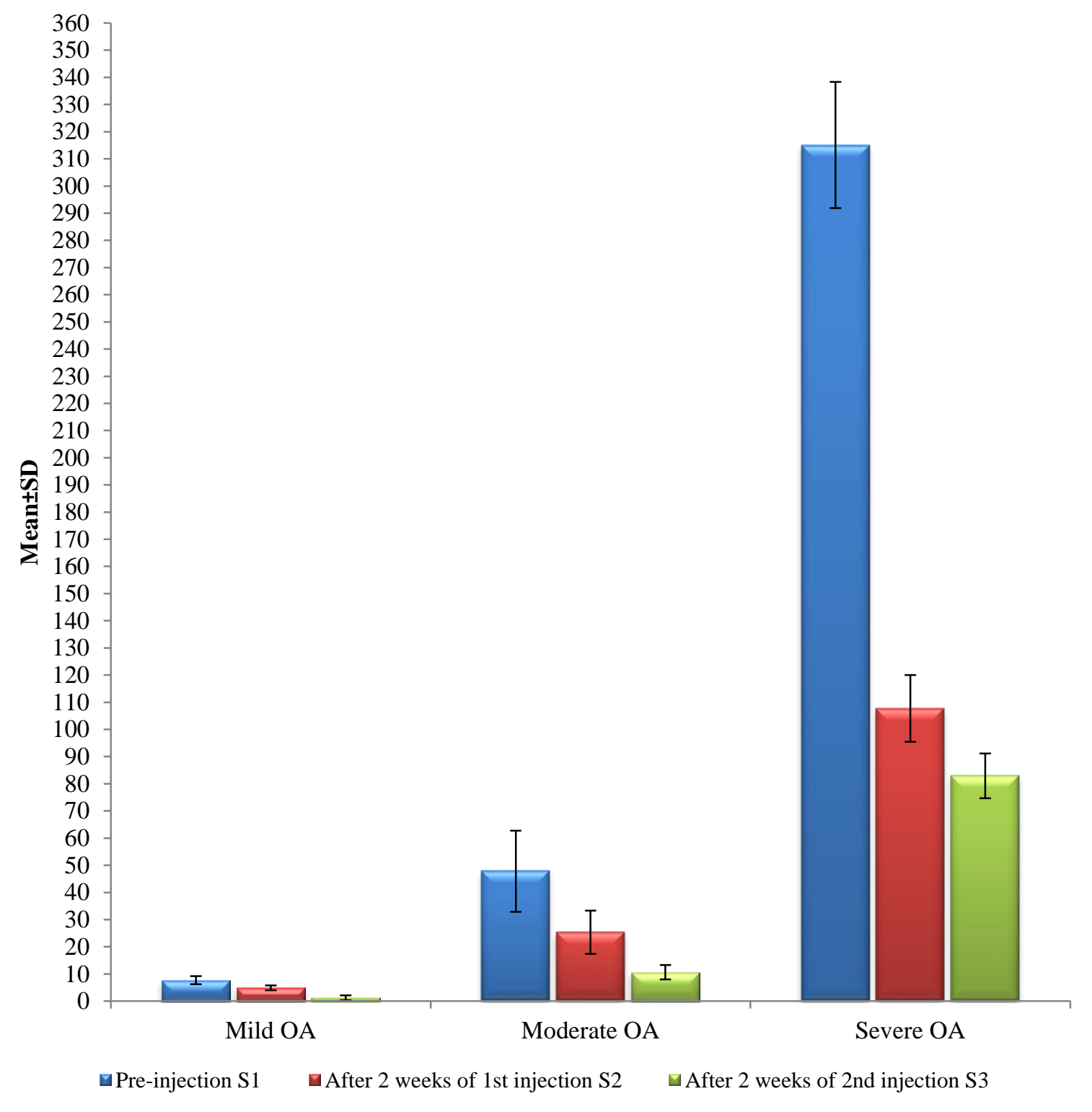

Fig. 2: Comparison of the mean synovial fluid levels of Tumor Necrosis Factor (TNF)- $\alpha$ (A) and Macrophage Migration Inhibitory Factor (MIF) (B) relative to the severity of knee Osteoarthritis (OA). ANOVA Post Hoc test (Fisher's LSD) was used.

Table 1: Demographic data of the included patients with knee osteoarthritis

\begin{tabular}{|c|c|c|c|c|c|c|c|c|c|c|c|}
\hline \multirow[b]{2}{*}{ Variables } & & \multirow[b]{2}{*}{$\begin{array}{l}\text { Total patients } \\
(\mathrm{n}=90,100 \%)\end{array}$} & \multirow{2}{*}{\multicolumn{2}{|c|}{$\begin{array}{l}\text { Mild OA } \\
(\mathrm{n}=30,33.3 \%)\end{array}$}} & \multirow{2}{*}{\multicolumn{2}{|c|}{$\begin{array}{l}\text { Moderate OA } \\
(\mathrm{n}=30,33.3 \%)\end{array}$}} & \multirow{2}{*}{\multicolumn{2}{|c|}{$\begin{array}{l}\text { Severe OA } \\
(\mathrm{n}=30,33.3 \%)\end{array}$}} & \multicolumn{3}{|c|}{$\mathrm{p}$-value } \\
\hline & & & & & & & & & $\mathrm{P} 1$ & $\mathrm{P} 2$ & $\mathrm{P} 3$ \\
\hline \multirow[t]{2}{*}{ Sex } & Male & $10 \%$ & 6 & $20 \%$ & 0 & $0 \%$ & 3 & $10 \%$ & 0.136 & 0.531 & 0.305 \\
\hline & Female & 90 & 24 & $80 \%$ & 30 & $100 \%$ & 27 & $90 \%$ & & & \\
\hline Age(years) & Mean \pm SD & $45 \pm 10.6$ & 46. & & $46 \pm$ & & 43. & & 0.871 & 0.517 & 0.626 \\
\hline Duration (months) & Mean \pm SD & $28.8 \pm 17.5$ & 33. & & 26.3 & & 26. & & 0.386 & 0.428 & 0.940 \\
\hline $\operatorname{BMI}\left(\mathrm{kg} / \mathrm{m}^{2}\right)$ & Mean \pm SD & $31.9 \pm 2.9$ & $31=$ & & 32.1 & & 32. & & 0.410 & 0.234 & 0.707 \\
\hline
\end{tabular}

p1 = mild Vs. moderate knee OA groups; p2 = mild vs. severe knee OA groups; 33 = moderate vs. severe OA groups. ANOVA and post-Hoc test (LSD: Least Significant Difference) was used as the data were normally distributed 
Table 2: Comparison of synovial fluid levels of TNF- $\alpha$ at different durations after the IA PRP injection in patients with knee OA

\begin{tabular}{|c|c|c|c|c|c|c|}
\hline \multirow{2}{*}{$\begin{array}{l}\text { TNF- } \alpha(\mathrm{pg} / \mathrm{mL}, \\
\text { Mean } \pm \text { SD) }\end{array}$} & \multirow[b]{2}{*}{$\mathrm{S} 1(\mathrm{n}=30)$} & \multirow[b]{2}{*}{$\mathrm{S} 2(\mathrm{n}=30)$} & \multirow[b]{2}{*}{$\mathrm{S} 3(\mathrm{n}=30)$} & \multicolumn{3}{|l|}{$\mathrm{P}$-value } \\
\hline & & & & $\mathrm{P} 1$ & $\mathrm{P} 2$ & $\mathrm{P} 3$ \\
\hline $\begin{array}{l}\text { Mild OA } \\
\text { (Min.-Max.) }\end{array}$ & $\begin{array}{l}35.5 \pm 7.4 \\
(27-44)\end{array}$ & $\begin{array}{l}17.8 \pm 7.5 \\
(6.25-30)\end{array}$ & $\begin{array}{l}9.3 \pm 5.4 \\
(4.5-13.56)\end{array}$ & $<0.001$ & $<0.001$ & 0.01 \\
\hline $\begin{array}{l}\text { Moderate OA } \\
\text { (Min.-Max.) }\end{array}$ & $\begin{array}{l}97.3 \pm 31.7 \\
(52-134)\end{array}$ & $\begin{array}{l}64.6 \pm 15.3 \\
(44-91)\end{array}$ & $\begin{array}{l}44.8 \pm 11.5 \\
(32-64)\end{array}$ & 0.002 & $<0.001$ & 0.048 \\
\hline $\begin{array}{l}\text { Severe OA } \\
\text { (Min.-Max.) }\end{array}$ & $\begin{array}{l}144.8 \pm 13.6 \\
(127-166.25)\end{array}$ & $\begin{array}{l}82.4 \pm 4.3 \\
(76-89)\end{array}$ & $\begin{array}{l}59.7 \pm 3.9 \\
(53.13-66)\end{array}$ & $<0.001$ & $<0.001$ & $<0.001$ \\
\hline
\end{tabular}

P1 = S1 vs. S2; P2 = S1 vs. S3; P3 = S2 vs. S3. ANOVA and post-Hoc test (LSD: Least Significant Difference) was used as the data were normally distributed

N.B: TNF- $\alpha$ : Tumor Necrosis Factor- $\alpha$; PRP: Platelet Rich Plasma; OA: Osteoarthritis; SD: Standard Deviation; S1: First synovial fluid sample assay (before IA injection of PRP); S2: Second synovial fluid sample assay (2 weeks after the first IA injection of PRP); S3: Third synovial fluid sample assay (2 weeks after the second IA injection of PRP); Min.: Minimum value; Max.: Maximum value

Table 3: Comparison of synovial fluid levels of MIF at different durations after the IA PRP injection in patients with knee OA

\begin{tabular}{|c|c|c|c|c|c|c|}
\hline \multirow{2}{*}{$\begin{array}{l}\text { MIF }(\mu \mathrm{g} / 1, \\
\text { Mean } \pm \mathrm{SD})\end{array}$} & \multirow[b]{2}{*}{$\mathrm{S} 1(\mathrm{n}=30)$} & \multirow[b]{2}{*}{$\mathrm{S} 2(\mathrm{n}=30)$} & \multirow[b]{2}{*}{$\mathrm{S} 3(\mathrm{n}=30)$} & \multicolumn{3}{|l|}{ P-value } \\
\hline & & & & P1 & $\mathrm{P} 2$ & P3 \\
\hline $\begin{array}{l}\text { Mild OA } \\
\text { (Min.-Max.) }\end{array}$ & $\begin{array}{l}7.7 \pm 1.5 \\
(5.5-10)\end{array}$ & $\begin{array}{l}4.9 \pm 0.9 \\
(4-6)\end{array}$ & $\begin{array}{l}1.25 \pm 0.9 \\
(0.25-3)\end{array}$ & $<0.001$ & $<0.001$ & $<0.001$ \\
\hline $\begin{array}{l}\text { Moderate OA } \\
\text { (Min.-Max.) }\end{array}$ & $\begin{array}{l}47.8 \pm 14.9 \\
(20.3-68)\end{array}$ & $\begin{array}{l}25.3 \pm 7.9 \\
(10.5-37.5)\end{array}$ & $\begin{array}{l}10.6 \pm 2.6 \\
(6.25-14.25)\end{array}$ & $<0.001$ & $<0.001$ & 0.003 \\
\hline $\begin{array}{l}\text { Severe OA } \\
\text { (Min.-Max.) }\end{array}$ & $\begin{array}{l}315.1 \pm 23.2 \\
(281-364)\end{array}$ & $\begin{array}{l}107.7 \pm 12.3 \\
(90-127)\end{array}$ & $\begin{array}{l}82.9 \pm 8.2 \\
(72.57-96.5)\end{array}$ & $<0.001$ & $<0.001$ & 0.002 \\
\hline
\end{tabular}

P1 = S1 vs. S2; P2 = S1 vs. S3; P3 = S2 vs. S3. ANOVA and post-Hoc test (LSD: Least Significant Difference) was used as the data were normally distributed

N.B: MIF: Macrophage Migration Inhibitory factor; PRP: Platelet Rich Plasma; OA: Osteoarthritis; SD: Standard Deviation; S1: First synovial fluid sample assay (before IA injection of PRP); S2: Second synovial fluid sample assay (2 weeks after the first IA injection of PRP); S3: Third synovial fluid sample assay (2 weeks after the second IA injection of PRP); Min.: Minimum value; Max.: Maximum value

The mean levels of synovial MIF were significantly higher (S1, pre-injection) among patients with severe OA $(315.1 \pm 23.2 \mu \mathrm{g} / \mathrm{L})$ than among those with mild $(7.7 \pm 1.5$ $\mu \mathrm{g} / \mathrm{L})$ or moderate OA $(47.8 \pm 14.9 \mu \mathrm{g} / \mathrm{L}), \mathrm{p}<0.05$ for all (Fig. 2B). Significantly lower synovial TNF- $\alpha$ levels were observed 2 weeks after the second IA injection of PRP (S3) in patients with mild, moderate, or severe knee OA $(1.25 \pm 0.9, \quad 10.6 \pm 2.6$ and $82.9 \pm 8.2 \mu \mathrm{g} / \mathrm{L}$, respectively) compared with pre-injection synovial fluid levels $(7.7 \pm 1.5$, $47.8 \pm 14.9$ and $315.1 \pm 23.2$, respectively) and levels 2 weeks after the first injection (S2) $(4.9 \pm 0.9,25.3 \pm 7.9$ and $107.7 \pm 12.3 \mu \mathrm{g} / \mathrm{L}$, respectively); $\mathrm{p}<0.05$ for all (Table 3 ).

Using Pearsons' correlation coefficient among patients with knee OA $(n=90)$, there was significant positive correlation between the synovial fluid TNF- $\alpha$ and MIF levels in $\mathrm{S} 1(\mathrm{r}=0.815, \mathrm{p}=0.000), \mathrm{S} 2(\mathrm{r}=0.783, \mathrm{p}=$ $0.000)$ and $S 3(r=0.750, p=0.000)$.

\section{Effects of IA Injection of PRP on Pain Score Among Patients with Knee OA}

Significantly lower median pain score values when assessed 2 weeks from the second IA injection in patients with mild, moderate or severe knee OA (2, 2 and 5, respectively) compared with the pre-injection pain scores (3, 6 and 9 , respectively), $\mathrm{p}<0.05$ for all (Table4).

\section{Therapeutic Effects of IA Injection of PRP on MOAKS in Patients with Knee OA}

Comparing MOAKS pre-injection value versus that two weeks from the second IA injection of PRP, there was significant improvement in bone marrow lesions among patients with only mild knee OA $(\mathrm{p}<0.05)$ and a significant improvement in synovitis in both patients with mild or moderate knee $\mathrm{OA}(\mathrm{p}<0.05$ for both), (Fig. 3A,3B and Table5).

There were no significant improvements in either patellofemoral cartilage volume or meniscal disintegrity in various severities of knee OA, p $>0.05$ for all (Fig. 3C and Table 5).

\section{Correlations of Pain Scores with Serial Synovial TNF- $\alpha$ and MIF Levels}

Using Pearsons' correlation coefficient, the included patients with knee OA $(n=90)$ showed significantly positive correlations between preinjection synovial fluid TNF- $\alpha$ levels (S1) and the preinjection pain score $(\mathrm{r}=0.849, \mathrm{p}=0.000)$, (Fig. 4A) and between synovial fluid TNF- $\alpha$ levels 2 weeks after the second injection (S3) and the post-injection pain score $(r=0.536, p=0.000)$, (Fig. 4B). 


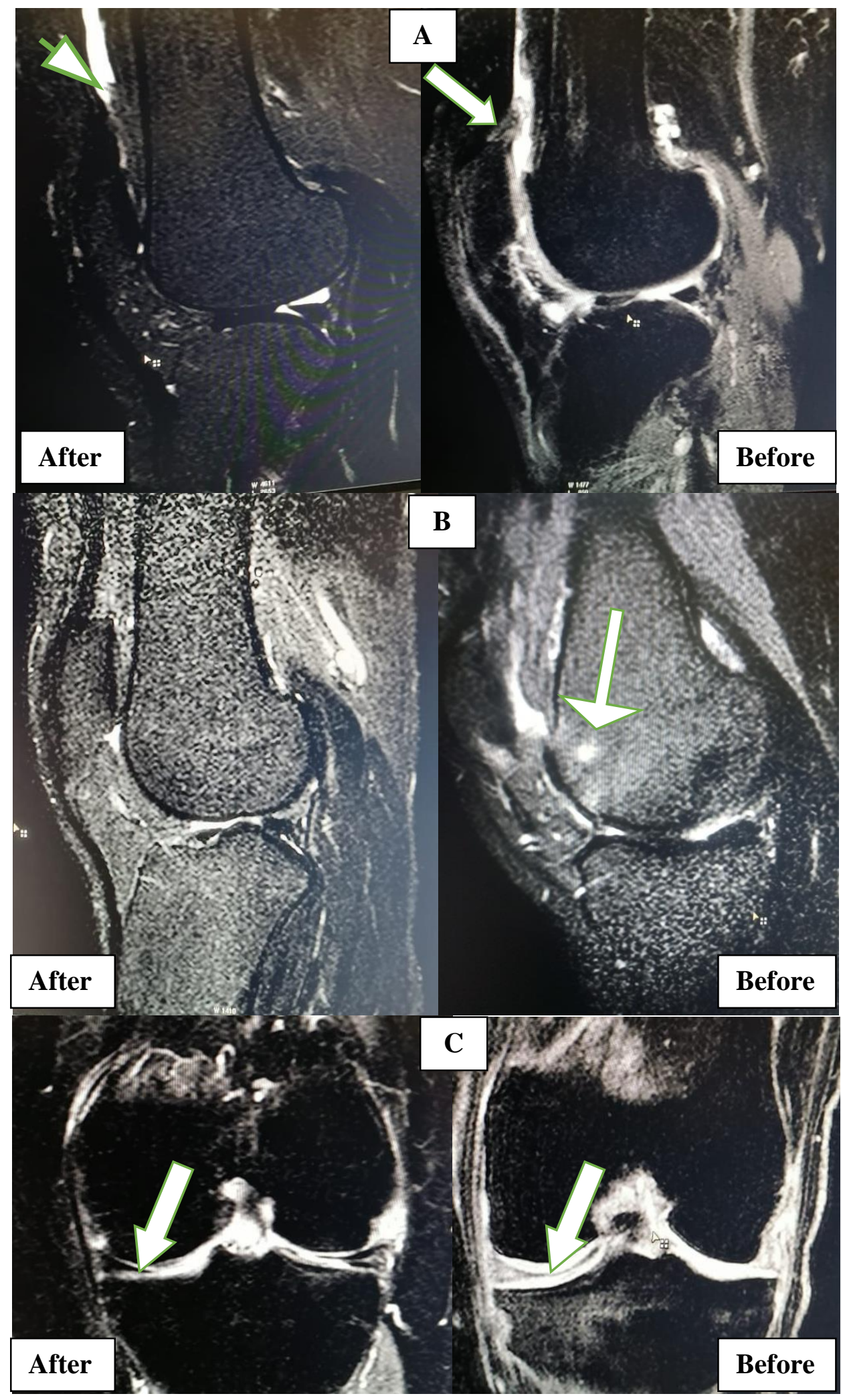

Fig. 3: MRI analysis of knee joint before the first IA injection of PRP and 2 weeks after the second IA PRP injection, MRI signals showed significant improvements in synovitis (A) and bone marrow lesions (B) with no improvement in cartilage loss(C) compared with the pre injection knee joint 

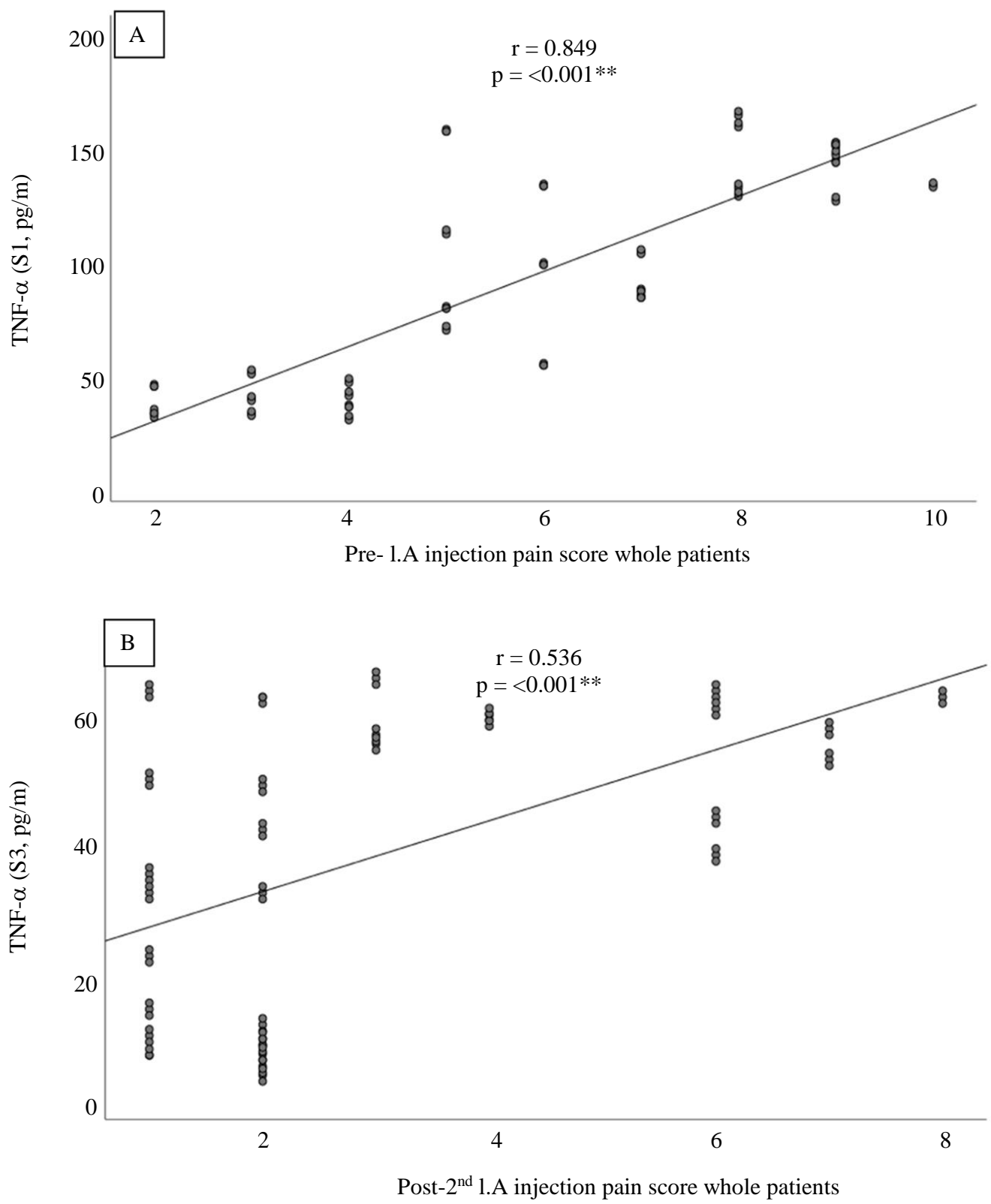

Fig. 4: Correlation of the pain score with synovial TNF- $\alpha$ levels in patients with knee osteoarthritis ( $\mathrm{n}=90)$ both pre-injection (A) and 2 weeks after the second injection (B). Pearson correlation was used

Table 4: Effects of the IA injection of PRP on pain score in patients with knee OA of varying severities

\begin{tabular}{|c|c|c|c|c|c|}
\hline \multirow[b]{2}{*}{ Patient groups } & & \multicolumn{2}{|l|}{ Pain score } & \multirow[b]{2}{*}{ MW* } & \multirow[b]{2}{*}{ p-value } \\
\hline & & Pre- injection & $\begin{array}{l}2 \text { weeks after the } \\
\text { second injection }\end{array}$ & & \\
\hline \multirow[t]{2}{*}{ Mild OA $(\mathrm{n}=30)$} & Median & 3 & 2 & 9 & 0.001 \\
\hline & IQR & $2-4$ & $1-2$ & & \\
\hline \multirow[t]{2}{*}{ Moderate OA $(\mathrm{n}=30)$} & Median & 6 & 2 & 11 & 0.002 \\
\hline & IQR & $5-7$ & $1-3$ & & \\
\hline \multirow[t]{2}{*}{ Severe OA $(\mathrm{n}=30)$} & Median & 9 & 5 & 2 & $<0.001$ \\
\hline & IQR & $8-9$ & $3-7$ & & \\
\hline
\end{tabular}

IA: Intra-Articular; PRP: Platelet-Rich Plasma; OA: Osteoarthritis IQR: Inter-Quartile Range; *MW: Mann-Whitney Test was used 

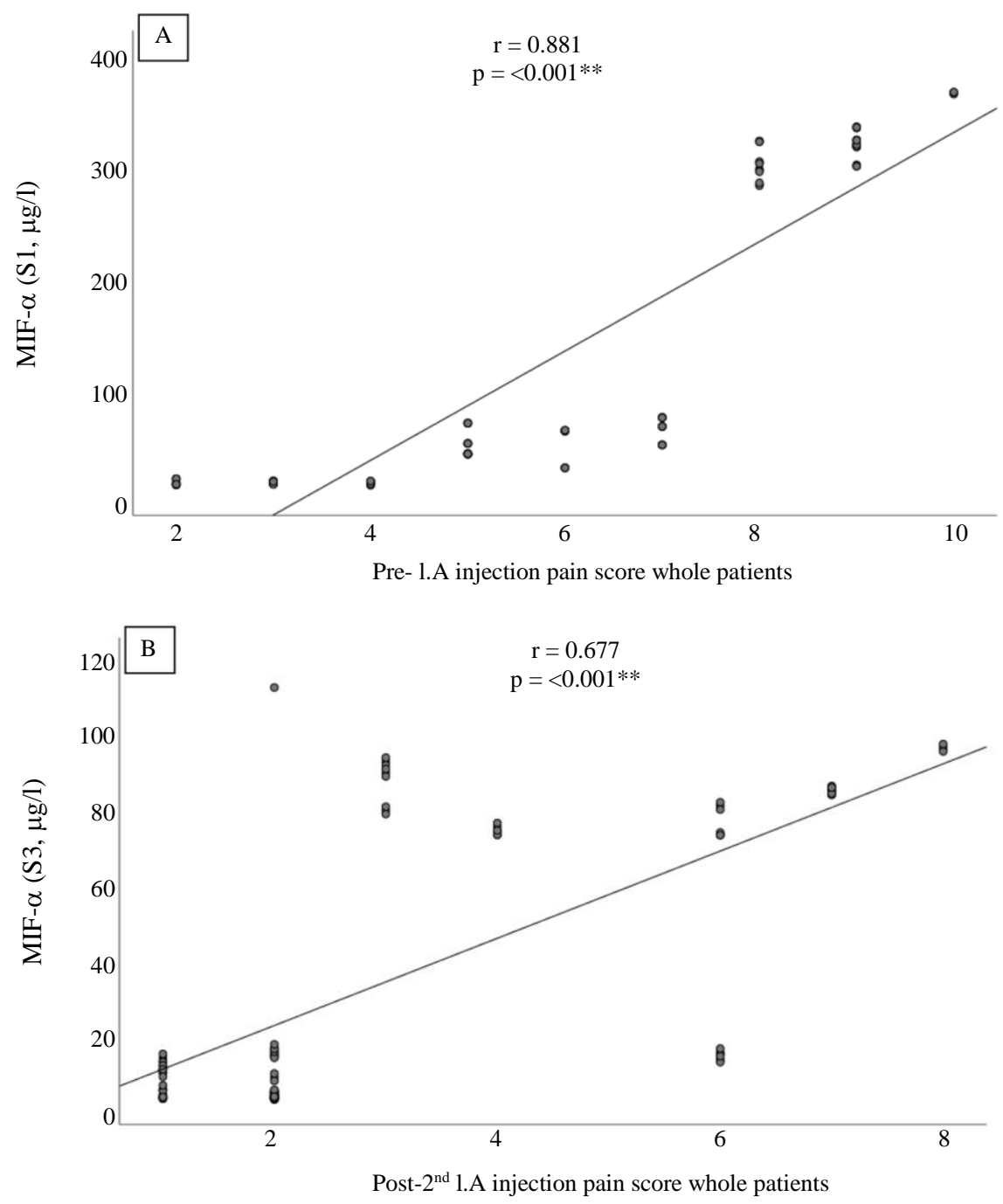

Fig. 5: Correlations of the pain score with synovial MIF levels in patients with knee osteoarthritis $(\mathrm{n}=90)$ both pre-injection $(\mathrm{A})$ and 2 weeks after the second injection (B). Pearson correlation was used

Table 5: Comparison of the therapeutic effects of the IA injection of PRP on MOAKS in patients with knee OA

\begin{tabular}{|c|c|c|c|}
\hline Patient groups & Pre- injection & 2 weeks after the second IA injection & P-value \\
\hline \multicolumn{4}{|c|}{ Patello-femoral cartilage volume (mean \pm SD) } \\
\hline Mild OA $(\mathrm{n}=30)$ & $0.24 \pm 0.04$ & $0.22 \pm 0.05$ & 0.227 \\
\hline Moderate OA $(\mathrm{n}=30)$ & $0.55 \pm 0.10$ & $0.55 \pm 0.10$ & 1.0 \\
\hline Severe OA $(\mathrm{n}=30)$ & $0.71 \pm 0.08$ & $0.70 \pm 0.08$ & 0.677 \\
\hline \multicolumn{4}{|c|}{ Meniscal disintegrity (mean \pm SD) } \\
\hline Mild OA $(\mathrm{n}=30)$ & $1.0 \pm 0.0$ & $1.0 \pm 0.0$ & ---- \\
\hline Moderate OA $(\mathrm{n}=30)$ & $2.0 \pm 0.0$ & $2.0 \pm 0.0$ & ---- \\
\hline Severe OA $(\mathrm{n}=30)$ & $3.0 \pm 0.0$ & $2.1 \pm 1.4$ & 0.280 \\
\hline \multicolumn{4}{|c|}{ Bone marrow abnormality (mean $\pm \mathrm{SD}$ ) } \\
\hline Mild OA & $0.30 \pm 0.02$ & $0.19 \pm 0.07$ & 0.001 \\
\hline Moderate OA & $0.45 \pm 0.13$ & $0.41 \pm 0.18$ & 0.684 \\
\hline Severe OA & $0.75 \pm 0.06$ & $0.73 \pm 0.06$ & 0.579 \\
\hline \multicolumn{4}{|l|}{ Synovitis $($ mean $\pm \mathrm{SD})$} \\
\hline Mild OA & $0.80 \pm 0.79$ & $0.02 \pm 0.01$ & 0.0393 \\
\hline Moderate OA & $1.80 \pm 0.91$ & $0.03 \pm 0.0$ & 0.001 \\
\hline Severe OA & $1.81 \pm 1.54$ & $0.03 \pm 0.0$ & 0.143 \\
\hline
\end{tabular}

N.B: I.A (Intra-Articular); OA (Osteoarthritis); PRP (Platelet-Rich Plasma); MOAKS: MRI Osteoarthritis Knee Score; SD: Standard deviation. Independent sample T-test was used 
Table 6: Comparison of the therapeutic outcomes of the IA injection of PRP in patients with varying severities of knee OA

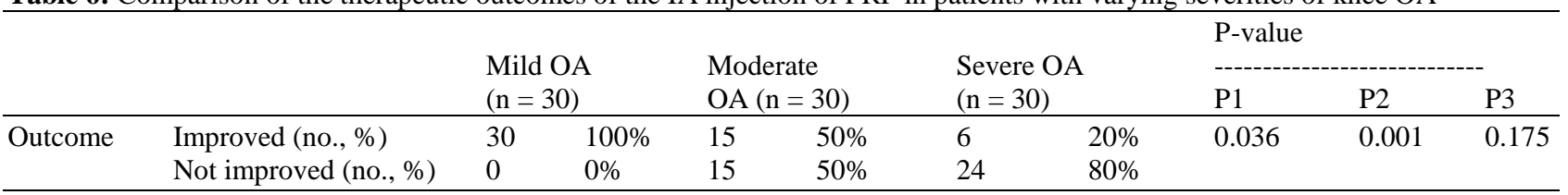

$\mathrm{P} 1=$ mild vs. moderate; $\mathrm{p} 2=$ mild vs. severe; $\mathrm{p} 3=$ moderate vs. severe. Chi-square test was used

Also, there were significantly positive correlations between pre-injection synovial fluid MIF levels (S1) and the pre-injection pain score $(\mathrm{r}=0.881, \mathrm{p}=0.000)$, (Fig. $5 \mathrm{~A}$ ) and between synovial fluid TNF- $\alpha$ levels 2 weeks after the second injection (S3) and the post-injection pain score $(r=0.677, p=0.000)$, (Fig. 5B).

\section{Therapeutic Outcomes of IA PRP Injection in Patients with Varying Severities of Knee OA}

Comparing the therapeutic efficacy of the IA PRP injection in patients with knee OA, there was improvement (indicated by lower VAS score) in all patients with mild knee OA (30 cases, 100\%), where as there were improvements in pain scores in 15 patients $(50 \%)$ with knee OA showed improvement and in only six patients (20\%) with severe knee OA (Table 6).

\section{Discussion}

Knee Osteoarthritis (OA) in subjects over 50 years is a major cause of pain and impairment and significantly affects physical activity and quality of life (Conrozier et al., 2019). The present study evaluated the therapeutic effects of IA PRP injection in patients with knee OA using pain score, MOAKS and biochemical assays of the synovial fluid cytokines TNF- $\alpha$ and MIF.

Obesity or being overweight has long been recognized as important risk factors for OA, particularly knee OA (Powell et al., 2005). This study included predominantly female subjects with female- to- male ratio 9:1. Moreover, there was higher frequency of patients categorized as obesity class 1 followed by obesity class 2 . Those categorized as overweight represented the least number of patients with knee OA. This corresponded with the findings of previous studies (Felson, 1990; Spector et al., 1994; Snijders et al., 2011; Hawamdeh and Al-Ajlouni, 2013; Zheng and Chen, 2015; Wallace et al., 2017). The reason for the predominance of females may be multifactorial and involves structural variations, prior trauma, genetic and hormonal disorders. Men had significantly higher total tibial and patella cartilage volumes than women. Women also had a significantly higher prevalence of baseline patellar cartilage defects. Over time females exhibited more volume loss in the knee cartilage than males (Hame and Alexander, 2013). One mechanism by which obesity leads to knee OA is the possibly increased mechanical load on the joint. Knee overloading may cause synovial joint breakdown and failure of ligaments and other structural support (Hame and Alexander, 2013).
Although PRP has been approved as an agent for knee OA therapy, studies of PRP in knee OA have found consistently more positive results than hyaluronic acid, other Intra-Articular (IA) injections and placebo compared with other musculoskeletal tissues (Dai et al., 2017; Huang et al., 2018 and O'Connell et al., 2019). Although the causes of knee OA are not understood completely, laboratory and clinical evidence suggests that inflammatory cytokines contribute to its pathogenesis (Chiu et al., 2011; Kapoor et al., 2011). The present study revealed significantly higher synovial fluid inflammatory cytokine (TNF- $\alpha$ and MIF) levels (S1, pre-injection) among patients with severe OA than in those with mild and moderate OA. Significantly lower synovial TNF- $\alpha$ and MIF levels were observed 2 weeks after the second IA injection of PRP (S3) in patients with mild, moderate or severe knee OA than levels from both the pre-injection synovial fluid and 2 weeks after the first injection (S2). These results indicate that PRP treatment for patients with knee OA yielded beneficial effects in regulating inflammatory factors. Inflammatory cytokines form a complex regulatory signaling network in femoral head osteonecrosis, which is mediated by different intracellular kinase signaling pathways to regulate the recruitment, stimulation and activation of autoimmune cells (Lebouvier et al., 2015). In accordance, Zhang et al. (2016) reported that MIF levels in synovial fluid, but not in serum, were associated independently with the severity of self-reported pain in patients with OA. Huang et al. (2018) reported significantly down regulated plasma concentrations of cytokines involving TNF- $\alpha$ after PRP treatment in patients with knee OA.

Our results revealed significantly positive correlations between synovial fluid TNF- $\alpha$ and MIF levels in patients with knee OA. MIF is a potent proinflammatory cytokine that causes the release of many inflammatory cytokines, Includinginterferon (IFN)- $\gamma$, Interleukin (IL)- $1 \beta, 6,8$ and TNF- $\alpha$ by initiating an inflammatory cascade (Leech et al.,1999).

We evaluated the therapeutic effects of IA PRP injections on the pain score and MOAKS in patients with knee OA. There were significantly lower pain score values when assessed 2 weeks after the second IA injection in patients with knee OA and significant improvements in synovitis and bone marrow lesions primarily for mild knee $\mathrm{OA}$ and to a lesser extent for moderate OA. Unfortunately, the treated patients did not show any significant improvements in the patella-femoral cartilage volume or meniscal disintegrity. Similarly, 
Burchard et al. (2019) suggested that the IA injection of PRP may improve symptoms of OA and decrease pain in patients with knee joint OA, independent of the level of cartilage damage as quantified by a whole organ MRI scoring method. Furthermore, a study by Laudy et al. (2015) demonstrated that in patients with knee OA, PRP injections resulted in decreased pain, improved function and global assessment and changes in joint imaging.

Favorable outcomes were observed in all patients with mild knee symptoms $(100 \%)$, in $50 \%$ of patients with moderate severity and $20 \%$ of patients with severe knee OA showed improvements in pain score. Consistent with our findings, Taniguchi et al. (2018) reported that IA PRP injection is probably a safe treatment option for Japanese patients with mild to moderate $\mathrm{OA}$ of the knee and may result in pain relief for up to 6 months. Although the restorative effects of PRP therapy on the cartilage are controversial, antiinflammatory effects, the down regulation of cytokine levels and joint homeostasis may explain the favorable effects in patients with severe OA (Kon et al., 2010; Marmotti et al., 2015).

Increasing evidence shows that the over expressed inflammatory cytokines in inflamed joints play an important pathophysiological role in generating and maintaining OA-induced pain by acting on nociceptive nerve cells (Penninx et al., 2004). We observed significantly positive correlations between synovial fluid TNF- $\alpha$ and MIF levels with the pain score. Consistent with our findings, Stannus et al. (2013) reported that TNF- $\alpha$ was associated positively with total knee worsening and pain.

\section{Conclusion}

The present study confirms the presence of inflammatory processes in the pathogenesis of knee OA including the inflammatory cytokines TNF- $\alpha$ and MIF. The anti-inflammatory effect is one of the therapeutic mechanisms that explains the efficacy of the IA PRP injection in patients with knee OA.PRP may lower the levels of synovial fluid cytokines (TNF- $\alpha$ and MIF), which subsequently improves pain and synovitis. This favorable outcome is achieved primarily in patients exhibiting mild $\mathrm{OA}$ and to a lesser extent moderate $\mathrm{OA}$. Therefore, this treatment represents a safe adjuvant biological therapy for these patients. We should noted that the synovial fluid cytokines' levels could change after a single injection over time and therefore the findings on the first week after a single injection may have differed over time and perhaps have continued to have decreased not requiring the addition of two more injections which require further researches.

\section{Study Limitations}

The lack of long-term follow up on the patients, which should be done in future studies and a small sample size were the main limitations of our study. Lack of control group was because of ethical concerns as it is difficult to take synovial fluid samples from healthy volunteers. Lack of characterization of the PRP samples that were injected, specifically, the platelet counts, the percentages of white cells, red blood cells and other cellular components within the PRP and the correlation of these counts to the findings of the various synovial fluid inflammatory markers that were obtained were also the other study limitations that will be approached in future related researches.

\section{Funding}

The research was partially funded by South Valley University, Faculty of Medicine, Qena 83523, Egypt.

\section{Authors' Contributions}

Mohammed H. Hassan: Study concept and design, blood sampling, biochemical and laboratory assays, PRP preparation, statistical analysis, literature research, first manauscript drafting.

Sawsan Abuhamdah: Statistical analysis, literature research.

Tahia H. Saleem: Literature research.

Elsayed Said: Study concept and design; clinical evaluation of the cases.

Nehal Ashraf Zaki: PRP preparation, Statistical analysis.

Ghada M. Abdelrazek: Radiological evaluation of patients, literature research, statistical analysis.

Safaa Y. Salim: Literature research, biochemical and laboratory assays, PRP preparation, statistical analysis.

Hamdy Tammam: Study concept and design; clinical evaluation of the cases.

\section{Availability of Data and Materials}

The datasets used and/or analyzed during the current study are presented within the manuscript.

\section{Ethics Approval and Consent to Participate}

Prior to start of the study, approval from the Ethics Committee of Faculty of Medicine, South Valley University, Qena, Egypt, was obtained. The ethical approval code (SVU-MED-MBC004-19-1). The study was carried out in accordance with the Declaration of Helsinki. Written informed consent was obtained from every patient. 


\section{Competing Interests}

The author(s) declared no potential conflicts of interest with respect to the research, authorship and/or publication of this article.

\section{Abbreviations}

OA: Osteoarthritis; TNF- $\alpha$ : Tumor Necrosis Factor- $\alpha$; MIF: Macrophage migration Inhibitory Factor; PRP: Platelet-Rich Plasma; I.A: Intra-Articular; ELISA: Enzyme Linked Immunosorbent Assay; BMI: Body Mass Index; VAS: Visual Analog Scale; MOAKS: MRI Osteoarthritis Knee Score.

\section{References}

Ayhan, E., Kesmezacar, H., \& Akgun, I. (2014). Intraarticular injections (corticosteroid, hyaluronic acid, platelet rich plasma) for the knee osteoarthritis. World Journal of Orthopedics, 5(3), 351. https://doi.org/10.5312/wjo.v5.i3.351

Burchard, R., Huflage, H., Soost, C., Richter, O., Bouillon, B., \& Graw, J. A. (2019). Efficiency of platelet-rich plasma therapy in knee osteoarthritis does not depend on level of cartilage damage. Journal of Orthopaedic Surgery and Research, 14(1), 153. https://doi.org/10.1186/s13018-019-1203-0

Chakkalakal, J. V., Jones, K. M., Basson, M. A., \& Brack, A. S. (2012). The aged niche disrupts muscle stem cell quiescence. Nature, 490(7420), 355-360. https://doi.org/10.1038/nature11438

Chiu, W. C., Lai, Y. P., \& Chou, M. Y. (2011). Humanization and characterization of an anti-human TNF- $\alpha$ murine monoclonal antibody. PloS One, 6(1), e16373. https://doi.org/10.1371/journal.pone.0016373

Conrozier, T., Eymard, F., Chouk, M., \& Chevalier, X. (2019). Impact of obesity, structural severity and their combination on the efficacy of viscosupplementation in patients with knee osteoarthritis. BMC Musculoskeletal Disorders, 20(1), 376. https://doi.org/10.1186/s12891-019-2748-0

Dai, W. L., Zhou, A. G., Zhang, H., \& Zhang, J. (2017). Efficacy of Platelet-Rich Plasma in the Treatment of Knee Osteoarthritis: A Meta-analysis of Randomized Controlled Trials. Arthroscopy: The journal of arthroscopic \& related surgery: Official publication of the Arthroscopy Association of North America and the International Arthroscopy Association, 33, 659-670.e1. https://doi.org/10.1016/j.arthro.2016.09.024

Dwyer, J.T., Melanson, K.J., Sriprachy-Anunt, U., Cross, P., \& Wilson, M. (2000). Dietary Treatment of Obesity. In: Feingold, K.R., Anawalt, B., Boyce, A., Chrousos, G., de Herder, W. W.,... \& Dhatariya,K. (Eds.), Endotext. South Dartmouth (MA):MDText.com,Inc. https://www.ncbi.nlm.nih.gov/books/NBK278991/
Felson, D. T. (1990, December). The epidemiology of knee osteoarthritis: Results from the Framingham Osteoarthritis Study. Seminars in Arthritis and Rheumatism, 20, 42-50. https://doi.org/10.1016/0049-0172(90)90046-I

Halpern, B., Chaudhury, S., Rodeo, S. A., Hayter, C., Bogner, E., Potter, H. G., \& Nguyen, J. (2013). Clinical and MRI outcomes after platelet-rich plasma treatment for knee osteoarthritis. Clinical journal of sport medicine: official Journal of the Canadian Academy of Sport Medicine, 23(3), 238-239. https://doi.org/10.1097/JSM.0b013e31827c3846

Hame, S. L., \& Alexander, R. A. (2013). Knee osteoarthritis in women. Current Reviews in Musculoskeletal Medicine, 6(2), 182-187. https://doi.org/10.1007/s12178-013-9164-0

Hawamdeh, M. Z., \& Al-Ajlouni, J. M. (2013). The clinical pattern of knee osteoarthritis in Jordan: a hospital based study. International Journal of MedicalSciences, 10(6),790795. https://doi.org/10.7150/ijms.5140

Huang, G., Hua, S., Yang, T., Ma, J., Yu, W., \& Chen, X. (2018). Platelet-rich plasma shows beneficial effects for patients with knee osteoarthritis by suppressing inflammatory factors. Experimental and Therapeutic Medicine, 15(3), 3096-3102. https://doi.org/10.3892/etm.2018.5794

Hunter, D. J., Guermazi, A., Lo, G. H., Grainger, A. J., Conaghan, P. G., Boudreau, R. M., \& Roemer, F. W. (2011). Evolution of semi-quantitative whole joint assessment of knee OA: MOAKS (MRI Osteoarthritis Knee Score). Osteoarthritis and Cartilage, 19(8), 9901002. https://doi.org/10.1016/j.joca.2011.05.004

Kapoor, M., Martel-Pelletier, J., Lajeunesse, D., Pelletier, J. P., \& Fahmi, H. (2011). Role of proinflammatory cytokines in the pathophysiology of osteoarthritis. Nature Reviews Rheumatology, 7(1), 33. https://doi.org/10.1038/nrrheum.2010.196

Katz, J., \& Melzack, R. (1999). Measurement of pain. Surgical Clinics of North America, 79(2), 231-252. https://doi.org/10.1016/S0039-6109(05)70381-9

Kennedy, M. I., Whitney, K., Evans, T., \& LaPrade, R. F. (2018). Platelet-rich plasma and cartilage repair. Current Reviews in Musculoskeletal Medicine, 11(4), 573-582. https://doi.org/10.1007/s12178-018-9516-X

Kon, E., Buda, R., Filardo, G., Di Martino, A., Timoncini, A., Cenacchi, A., \& Marcacci, M. (2010). Plateletrich plasma: Intra-articular knee injections produced favorable results on degenerative cartilage lesions. Knee Surgery, Sports Traumatology, Arthroscopy, 18(4), 472-479. https://doi.org/10.1007/s00167-009-0940-8 
Laudy, A. B., Bakker, E. W., Rekers, M., \& Moen, M. H. (2015). Efficacy of platelet-rich plasma injections in osteoarthritis of the knee: A systematic review and meta-analysis. British Journal of Sports Medicine, 49(10), 657-672. https://doi.org/10.1136/bjsports2014-094036

Lebouvier, A., Poignard, A., Cavet, M., Amiaud, J., Leotot, J., Hernigou, P., Rahmouni, A., Bierling, P., Layrolle, P., Rouard, H., \& Chevallier, N. (2015). Development of a simple procedure for the treatment of femoral head osteonecrosis with intra-osseous injection of bone marrow mesenchymal stromal cells: study of their biodistribution in the early time points after injection. Stem Cell Research \&Therapy, 6(1), 68. https://doi.org/10.1186/s13287-015-0036-y

Leech, M., Metz, C., Hall, P., Hutchinson, P., Gianis, K., Smith, M., Weedon, H., Holdsworth, S. R., Bucala, R., \& Morand, E. F. (1999). Macrophage migration inhibitory factor in rheumatoid arthritis: evidence of proinflammatory function and regulation by glucocorticoids. Arthritis and Rheumatism, 42(8), 1601-1608. https://doi.org/10.1002/15290131(199908)42:8<1601::AID-ANR6>3.0.CO;2-B

Li, H., Xie, S., Qi, Y., Li, H., Zhang, R., \& Lian, Y. (2018). TNF- $\alpha$ increases the expression of inflammatory factors in synovial fibroblasts by inhibiting the PI3K/AKT pathway in a rat model of monosodium iodoacetate-induced osteoarthritis. Experimental and Therapeutic Medicine, 16(6), 4737-4744. https://doi.org/10.3892/etm.2018.6770

Llamas-Covarrubias, M. A., Valle, Y., Bucala, R., NavarroHernández, R. E., Palafox-Sánchez, C. A., PadillaGutiérrez, J. R., ... \& Muñoz-Valle, J. F. (2013). Macrophage migration inhibitory factor (MIF): genetic evidence for participation in early onset and early stage rheumatoid arthritis. Cytokine, 61(3), 759-765. https://doi.org/10.1016/j.cyto.2012.12.032

Marmotti, A., Rossi, R., Castoldi, F., Roveda, E., Michielon, G., \& Peretti, G. M. (2015). PRP and articular cartilage: A clinical update. BioMed Research International 2015, 542-502. https://doi.org/10.1155/2015/542502

Nurden, A. T. (2011). Platelets, inflammation and tissue regeneration. Thrombosis and Haemostasis, 105(S 06), S13-S33. https://doi.org/10.1160/THS10-11-0720

O'Connell, B., Wragg, N. M., \& Wilson, S. L. (2019). The use of PRP injections in the management of knee osteoarthritis. Cell and Tissue Research, 376(2), 143-152. https://doi.org/10.1007/s00441-019-02996-X

Penninx, B. W., Abbas, H., Ambrosius, W., Nicklas, B. J., Davis, C., Messier, S. P., \& Pahor, M. (2004). Inflammatory markers and physical function among older adults with knee osteoarthritis. The Journal of Rheumatology, 31(10), 2027-2031. https://www.jrheum.org/content/31/10/2027.short
Powell, A., Teichtahl, A. J., Wluka, A. E., \& Cicuttini, F. M. (2005). Obesity: a preventable risk factor for large joint osteoarthritis which may act through biomechanical factors. British Journal of Sports Medicine, 39(1), 4-5. https://doi.org/10.1136/bjsm.2004.011841

Pritzker, K. P., Gay, S., Jimenez, S. A., Ostergaard, K., Pelletier, J. P., Revell, P. A., Salter, D., \& van den Berg, W. B. (2006). Osteoarthritis cartilage histopathology: grading and staging. Osteoarthritis and Cartilage, 14(1), 13-29. https://doi.org/10.1016/j.joca.2005.07.014

Richebé, P., Capdevila, X., \& Rivat, C. (2018). Persistent Postsurgical Pain: Pathophysiology and Preventative Pharmacologic Considerations. Anesthesiology, 129(3), 590-607. https://doi.org/10.1097/ALN.0000000000002238

Rogers, J., \& Dieppe, P. (2003). Paleopathology of osteoarthritis. In: Brandt, K. D., Doherty, M.,\& Lohmander, L. S. (Eds.), Osteoarthritis, (pp. 57-65), Oxford: Oxford University Press.

Sampson, S., Reed, M., Silvers, H., Meng, M., \& Mandelbaum, B. (2010). Injection of platelet-rich plasma in patients with primary and secondary knee osteoarthritis: a pilot study. American Journal of Physical Medicine \&Rehabilitation, 89(12), 961-969. https://doi.org/10.1097/PHM.0b013e3181fc7edf

Snijders, G. F., den Broeder, A. A., van Riel, P. L. C. M., Straten, V. H. H. P., de Man, F. H. R., van den Hoogen, F. H. J., ... \& NOAC Study Group. (2011). Evidence-based tailored conservative treatment of knee and hip osteoarthritis: Between knowing and doing. Scandinavian Journal of Rheumatology, 40(3), 225-231. https://doi.org/10.3109/03009742.2010.530611

Spector, T. D., Hart, D. J., \& Doyle, D. V. (1994). Incidence and progression of osteoarthritis in women with unilateral knee disease in the general population: The effect of obesity. Annals of the Rheumatic Diseases, 53(9), 565-568. https://doi.org/10.1136/ard.53.9.565

Stannus, O. P., Jones, G., Blizzard, L., Cicuttini, F. M., \& Ding, C. (2013). Associations between serum levels of inflammatory markers and change in knee pain over 5 years in older adults: A prospective cohort study. Annals of the Rheumatic Diseases, 72(4), 535-540. https://doi.org/10.1136/annrheumdis-2011201047

Taniguchi, Y., Yoshioka, T., Kanamori, A., Aoto, K., Sugaya, H., \& Yamazaki, M. (2018). Intra-articular Platelet-Rich Plasma (PRP) injections for treating knee pain associated with osteoarthritis of the knee in the Japanese population: A phase I and IIa clinical trial. Nagoya journal of medical science, 80(1), 39-51. https://doi.org/10.18999/nagjms.80.1.39 
Wallace, I. J., Worthington, S., Felson, D. T., Jurmain, R. D., Wren, K. T., Maijanen, H., ... \& Lieberman, D. E. (2017). Knee osteoarthritis has doubled in prevalence since the mid-20th century. Proceedings of the National Academy of Sciences, 114(35), 9332-9336. https://doi.org/10.1073/pnas.1703856114

Zhang, P. L., Liu, J., Xu, L., Sun, Y., \& Sun, X. C. (2016). Synovial fluid macrophage migration inhibitory factor levels correlate with severity of self-reported pain in knee osteoarthritis patients. Medical science monitor: International medical journal of experimental and clinical research, 22, 2182-2186. https://doi.org/10.12659/MSM.895704
Zheng, H., \& Chen, C. (2015). Body mass index and risk of knee osteoarthritis: Systematic review and meta-analysis of prospective studies. BMJ Open, 5(12), e007568.

https://doi.org/10.1136/bmjopen-2014-007568 